\title{
Topology Correction Using Fast Marching Methods and Its Application to Brain Segmentation
}

\author{
Pierre-Louis Bazin and Dzung L. Pham \\ Johns Hopkins University, Baltimore, USA
}

\begin{abstract}
We present here a new method for correcting the topology of objects segmented from medical images. Whereas previous techniques alter a surface obtained from the hard segmentation of the object, our technique works directly in the image domain, propagating the topology for all isosurfaces of the object. From an analysis of topological changes and critical points in implicit surfaces, we introduce a topology progagation algorithm that enforces any desired topology using a fast marching technique. Compared to previous topology correction techniques, the method successfully corrects topology while effecting fewer changes to the original volume.
\end{abstract}

\section{Introduction}

The topological properties of two-dimensional (2D) and three-dimensional (3D) objects are often very simple, regardless of the complexity of the geometric object. The cortex of the human brain is a striking example; despite its intricate folds, it is considered to have the topology of a sphere, without any holes or handle-like junctions. Most organs and sub-structures found in the human body also share this spherical topology.

Ideally, algorithms that extract objects from $2 \mathrm{D}$ or $3 \mathrm{D}$ images should respect the object topology. A major problem is that topology is a global property of the object, whereas most extraction techniques operate locally on the pixels or voxels of an image. One solution is to start from an object with the desired topology and deform it on the image to follow the shape of the object to extract [10/7/3]. However, surface segmentation algorithms that constrain the topology also require an initialization close to the object of interest. Correcting the topology of objects is therefore often necessary, either before or after the surface extraction from an initial segmentation. This initial segmentation is typically the result of a fuzzy or statistical classification algorithm [911]) that computes a normalized membership value for each class and each pixel that varies continuously between zero and one.

Current algorithms for topology correction, however, operate on a binary volume extracted from the classification of the image data [16 13]6 15], generally obtained by performing a threshold at the 0.5 level set of the membership function for the class. Two types of techniques can be found in the literature: graph-based analysis and correction [6 15], and distance function processing, inspired by level set methods [16 13]. In places where changes are needed to enforce the spherical topology, these methods must decide whether to cut a handle or fill a hole, and where to proceed, based solely on the geometry of the original surface. In brain segmentation applications, the binary volumes also require pre-procesing to remove isolated parts and close holes [166[15]. 
Graph-based techniques convert the objects into graphs using either the 2D slices or a morphological opening to isolate sub-structures. The graph is then processed to identify cycles and remove them from the volume. The complexity of the analysis increases rapidly with the object size, and problems can occur at unusual configurations. Level set methods compute a distance function to encode the object shape, which changes when the volume is edited. In [13], candidate regions for addition or removal are identified and ranked through their distance function, so they must be recomputed whenever the object is modified and there is no guaranteed convergence. In all these methods, the intensity information available in the original image or in the membership function is largely ignored. If the object is highly convoluted, it may happen that the smallest change with regard to the geometry of the binary volume corresponds to including points with very low membership, or discarding points with very high membership.

In this work, we propose a new topology correction algorithm that can act directly on the membership function instead of the binary segmentation. The method propagates rigorous topological constraints on scalar 2D and 3D functions, even in the presence of noise. With a single computation of a modified fast marching method, the topology of the entire image is corrected. All isosurfaces extracted from the membership function or volumetric data will have the same topology, and we can even enforce non-spherical topologies, given an appropriate initialization. By working on the continuously-valued classification data rather than the binary segmentation, the amount of change effected by the topology correction is substantially reduced. The proposed method also provides greater flexibility in defining a desired surface. In some applications, isosurfaces at values other than 0.5 can be desirable [17]. Previous methods would require a separate topology correction for every desired isosurface value.

The paper is organized as follows. In Section 2, we study the topological properties of scalar fields and present a topology-preserving approximation algorithm for distance functions and membership functions. Section 3 details the topology correction algorithm, and Section 4 demonstrates its application to magnetic resonance brain images.

\section{Topological Properties of Scalar Fields}

Consider the general problem of extracting an object with a given topology from an image $I$ in $\mathbb{R}^{3}$. The object is represented as a volume $V$ bounded by a surface $S$, defined as the zero level set of a signed distance function $d: S=\left\{x \in \mathbb{R}^{3} \mid d(x)=0\right\}$. The image itself is a function of the object, and can be a distance function, membership function, or some other function. The image and the distance function are digital, so the values of $x$ are defined on a regular grid of voxels. The topology of the surface is characterized globally by its Euler Number. This measure, however, does not account for the type, location or extent of local topology changes.

In scalar fields, topology changes occur only at critical points [818]. These points are the singular points of the implicit isosurfaces, an extension of the non-simple points of binary images[2|4/7]. They have been classified in [18] as regular, flat, minimal, maximal and saddle points, either local or extended. At any non-regular point or region made of extended non-regular points, topology changes may occur when the isovalue used to construct the surface is equal to the value at that point. 
If the surface is moved away from its zero level set, it will keep the same topology until it reaches the value of a critical point. The changes are difficult to predict without a fine analysis of the critical point type (e.g. saddle points can have different effects). The only simple way to keep the topology invariant is to remove all critical points.

To identify critical points, consider the distance function $d(x)$ at a point $x$. We define positive, negative and equal regions to be connected regions of neighboring points $y$ with $d(y)>d(x), d(y)<d(x), d(y)=d(x)$, respectively). The neighborhood of a point is defined as the set of 6,18 or 26-connected neighbors on the digital grid of $x$ values. The numbers $N_{p}, N_{n}$ and $N_{e}$ of positive, negative and equal regions within a neighborhood determines the type of the point (see [1] for more details). The choice of connectivity will affect the shape of the object, as we will detail later. It is enough for the subsequent analysis to know if a point is regular or critical:

$$
x \text { is regular } \equiv N_{p}(x)=N_{n}(x)=1, N_{e}(x)=0
$$

Regions of equal value are a practical problem, as globally regular or critical regions could have the same $N_{p}, N_{n}$ and $N_{e}$ in a small neighborhood (e.g. subsample the grid by a factor 2: all points have equal neighbors and become critical). We can remove that problem by grouping equal points within either positive or negative regions.

\subsection{Topology-Preserving Distance Functions}

To compute the distance function $d(x)$ efficiently, we rely on a fast marching method, implemented through a binary tree sorting technique [14]: all points outside of the initial surface $S_{0}$ are ranked into a binary tree depending on their distance $d(X)$ to the surface (initially, the distances are all equal to 1 , but will increase as the algorithm evolves), then the surface is brought in front of the first of those points. Its neighbors (now in front of the surface) are added to the binary tree with an increased distance value, and the surface is moved again until the tree is empty. This method produces a distance function $d(x)$ accurate up to the grid scale, and the binary tree implementation provides $n \log n$ speed in the image size. To obtain a complete distance function, distances are propagated inside as well as outside $S_{0}$, with an inverse sign. With this distance function, arbitrary changes in the isosurface topology will occur when the isovalue is changed: parts will split or merge, holes will appear or disappear, etc (see Fig 1-b).

Using the previous analysis, we construct a modified distance function that will guarantee that the topology of the isosurface stays unchanged for all isovalues. The central idea is to detect critical points and change their distance while propagating the fast marching algorithm:

\section{Algorithm: Topology-preserving distance function}

1. starting from volume $V_{k}$ bounded by the surface $S_{k}$,

2. insert the points $x$ on the outside boundary $S_{k}$ of the volume $V_{k}$ in the tree, ranked by their distance $d(x)$ (at first, $d(x)=1$ ),

3. extract the first point $x$ from the tree,

4. if the point has been previously labeled as critical, its distance function becomes $d(x)=\max \{d(y)\}$, for $y$ inside the object and neighbors of $x$, otherwise $d(x)$

5. compute the number of positive and negative neighbors for $x$, 
6. if $x$ is regular, insert $x$ into the volume and insert its neighbors outside the volume, regular or critical, into the tree with their distance $d(y)=d(x)+1$

7. else, label $x$ as critical and set it aside,

8. go back to step 3, until the tree is empty.

This algorithm produces the regular distance function when the topology is invariant. Critical points are kept outside the volume until the neighboring distances make them regular: the algorithm not only isolates critical points but computes the exact amount of change needed to keep the volume regular while including them. This is illustrated in Figure 1 $\mathrm{c}$ : when the isovalue is low, the fingers stay connected from the bottom to the tip, until the distance is high enough in the middle to remove the finger completely. In the original geometric distance function, they split into several pieces before they disappear.
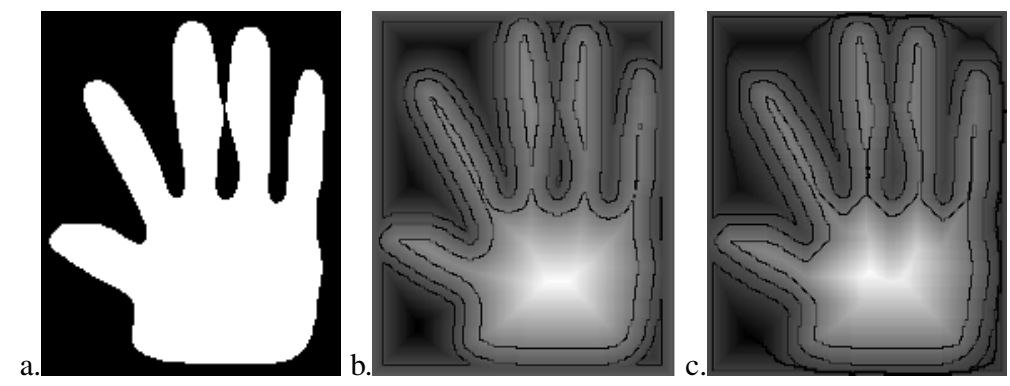

Fig. 1. Distance functions: a) the outline of a hand, b) the regular distance function started from the hand contour with three isocontours outlined, c) the topological distance function, with the same outlined isocontours. Note how the topology is different for the three regular isocontours. The topological distance function includes stronger skeleton-like features in the areas changed to maintain the correct topology, and all its isocontours have the same topology.

In the propagation, the object and background connectivity is controlled in step 5, when computing the number of positive and negative neighbors. If $N_{p}, N_{n}$ are computed with $6 / 26$ (resp. $6 / 18,18 / 6,26 / 6$ ) connectivity, then the volume and background are $6 / 26,6 / 18,18 / 6$ or $26 / 6$ connected.

\subsection{Topology-Preserving Membership Functions}

We can extend the topology propagation from distance functions to general scalar fields. We replace the distance function $d(x)$ with a scalar field $f(x)$ to be approximated by a function $g(x)$. The same propagation algorithm applies to $g$ :

\section{Algorithm: Topology propagation on scalar fields}

1. start from $g(x)=f(x)$ everywhere,

2. consider the volume $V_{k}$ bounded by $S_{k}:\left\{x \mid g(x)<=g_{k}\right\}$,

3. insert the points $x$ on the boundary (and outside) of the volume $V_{k}$ in the tree, ranked by their value $g(x)$,

4. extract the first point $x$ from the tree, 
5. if the point has not been labeled as critical, set the value as $f(x)$,

6. else, the field value becomes $g(x)=\max \{g(y)\}$ for $y$ inside $V_{k}$ and neighbor of $x$,

7. compute the number of positive and negative neighbors for $x$,

8. if $x$ is regular, insert $x$ into the volume (set $g_{k}=g(x)$ ) and insert its neighbors outside the volume or critical into the tree,

9. else, label $x$ as critical and set it aside,

10. go back to step 2, until the tree is empty.

This algorithm produces a scalar field as close as possible to $f(x)$, with the same topology as the starting volume $V_{0}$. As the changes are propagated along successive isovalues of the image, the technique is guaranteed to succeed in enforcing the original topology of $V_{0}$ in every isosurface of $g(x)$.

\section{Topology Correction Algorithm}

Topology correction aims at enforcing a chosen topology on the objects we want to extract. The correct or desired topology is known a priori, and depends on the application. Unlike previous techniques, our topology propagation technique will not just enforce spherical topology, but propagate through the distance or membership function any prior topology template. For spherical topology, a single point is enough to impose the sphericality constraint. For other topologies, different starting shapes can be used: a closed circle for doughnut or cylinder-like structures, a carved sphere for a hollow object, etc. Once we have chosen the appropriate template, we need to place it inside the object of interest. In the case of non-spherical topology, this step can be difficult as the holes and handles of the template should match those of the image. For spherical topology however, we just need to find a point inside the main object of interest. Starting from this point, we propagate the topology down from the highest membership values to the lowest:

\section{Algorithm: Spherical topology correction}

1. threshold the membership function at the 0.5 isovalue, and select the largest connected region,

2. in this region, select one of the points of highest membership value,

3. from this original point in the membership function, use the topology propagation algorithm toward lower membership values, with proper connectivity.

The initialization step ensures that we select a point inside the main structure of interest, except when dealing with several structures of similar size (in such case, it is necessary to select a point for each structure). The topology correction could as easily have started from a bounding box outside the image and converged toward the structure. From our experiments, we found that there is often more noise and outliers in the background area than holes in the high membership regions, and propagating the topology from the background would result in slightly more noise. This choice is consistent with the previous binary techniques that would only include the largest connected component. The corrected image with our algorithm is "included" in the original image: all corrected values can only be inferior or equal to the original ones. Methods to combine both directions of change are non-trivial, and would require to re-evaluate the topology at every change. 


\section{Experiments}

We have gathered a set of 10 brains processed for the Baltimore Longitudinal Study on Aging [12] to test and validate the topology correction algorithm. These brains were first stripped, then segmented into gray matter, white matter and CSF, and the white matter memberships were further edited to fill the sub-cortical area [5]. The images all have a $1 \mathrm{~mm}$ cubic resolution. We then perform the topology correction on the edited white matter memberships. The chosen connectivity is $18 / 6$, so that we could compare with previous topology correction techniques evaluated on similar data [15]6].

In all cases, the topology correction succeeds. Even though the topology of every isovalue is guaranteed to be spherical, we verified it by automated graph-based analysis [15]. The corrected membership functions are very close to the original ones (cf. Table 1): the number of changed voxels over the entire image is around $10 \%$, and the mean amount of change for these voxels (counting only changed voxels) is below 0.05 (the membership intensities are in $[0,1]$ ). The changes counted on the 0.5 isosurfaces are similar to those of previous methods, as shown in Table 2 We obtained results from the GTCA method [6] for the same data, and compared them to our 0.5 isovalues. Even though the proposed method enforces the topology on all isovalues, it causes

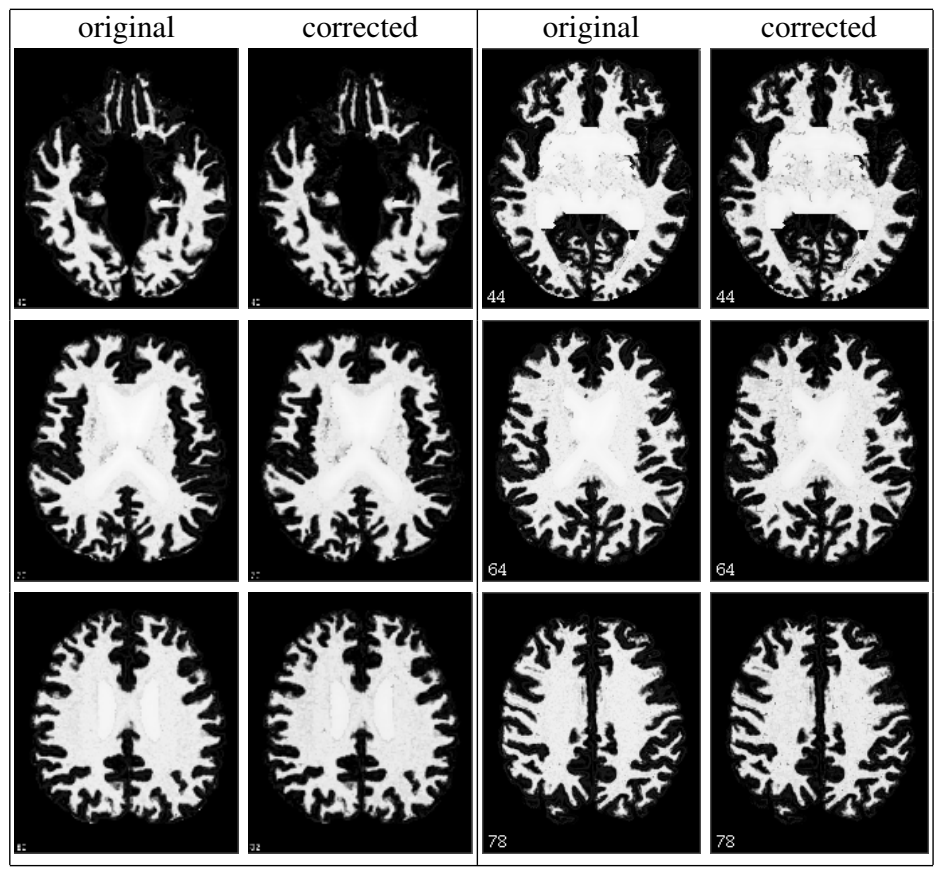

Fig. 2. Two examples of topology correction (brains number 4 and 7 of the experiments): three slices of the white matter membership function after filling of the internal parts, before and after the topology correction. Note the few visible changes: parts with high membership but disconnected from the white matter are removed, loops are cut (see top slices) and some patterns appear in the filled region due to remaining noise. 
Table 1. Topology correction on a set of brains

\begin{tabular}{|c|c|c|c|c|c|}
\hline $\begin{array}{c}\text { Brain } \\
\text { number }\end{array}$ & $\begin{array}{c}\text { Computation } \\
\text { time }\end{array}$ & $\begin{array}{c}\text { Total } \\
\text { voxels }\left(\mathrm{x} 10^{3}\right)\end{array}$ & $\begin{array}{c}\text { Changed } \\
\text { voxels }\left(\mathrm{x} 10^{3}\right)\end{array}$ & $\begin{array}{c}\% \text { of changed } \\
\text { voxels }\end{array}$ & $\begin{array}{c}\text { mean value } \\
\text { of change }\end{array}$ \\
\hline 1 & $52 \mathrm{~s}$ & 2,997 & 334 & 0.111 & 0.0294 \\
2 & $63 \mathrm{~s}$ & 3,370 & 412 & 0.122 & 0.0456 \\
3 & $53 \mathrm{~s}$ & 3,153 & 352 & 0.112 & 0.0471 \\
4 & $63 \mathrm{~s}$ & 3,654 & 409 & 0.112 & 0.0400 \\
5 & $59 \mathrm{~s}$ & 3,371 & 387 & 0.115 & 0.0446 \\
6 & $58 \mathrm{~s}$ & 3,407 & 383 & 0.113 & 0.0498 \\
7 & $65 \mathrm{~s}$ & 3,449 & 406 & 0.118 & 0.0432 \\
8 & $63 \mathrm{~s}$ & 3,425 & 416 & 0.121 & 0.0465 \\
9 & $69 \mathrm{~s}$ & 3,969 & 467 & 0.118 & 0.0448 \\
10 & $48 \mathrm{~s}$ & 2,862 & 316 & 0.110 & 0.0365 \\
\hline
\end{tabular}

Table 2. Topology correction on a set of brains: extracted 0.5 isovalue

\begin{tabular}{|c|c|c|c|c|c|c|c|c|}
\hline $\begin{array}{l}\text { Brain } \\
\text { number }\end{array}$ & \begin{tabular}{|l|l|} 
Initial Topology \\
FP FL BP BL
\end{tabular} & $\begin{array}{l}\text { Final To } \\
\text { FP FL B }\end{array}$ & $\begin{array}{l}\text { opol } \\
\text { BP }\end{array}$ & $\begin{array}{l}\text { ogy } \\
\text { BL }\end{array}$ & \begin{tabular}{|c|} 
Initial \\
brain size
\end{tabular} & $\begin{array}{c}\text { Changed } \\
\text { voxels }\end{array}$ & $\begin{array}{l}\% \text { of brain } \\
\text { changed }\end{array}$ & $\begin{array}{l}\% \text { of change } \\
\text { with GTCA }\end{array}$ \\
\hline 1 & $\begin{array}{|llll|}243 & 125 & 20 & 86\end{array}$ & \begin{tabular}{|ll}
1 & 0 \\
\end{tabular} & 1 & 0 & 607,236 & 1,422 & 0.234 & 0.190 \\
\hline 2 & $\begin{array}{llll}368 & 177 & 136 & 112\end{array}$ & 10 & 1 & 0 & 662,357 & 1,964 & 0.297 & 0.253 \\
\hline 3 & 464380207220 & 10 & 1 & 0 & 636,077 & 2,650 & 0.417 & 0.308 \\
\hline 4 & $28101144150 \quad 81$ & 10 & 1 & 0 & 708,954 & 1,279 & 0.180 & 0.171 \\
\hline 5 & $\begin{array}{llll}373 & 171 & 177 & 149\end{array}$ & 10 & 1 & 0 & 632,431 & 1,761 & 0.278 & 0.237 \\
\hline 6 & $\begin{array}{llll}441 & 218 & 46 & 174\end{array}$ & 10 & 1 & 0 & 594,337 & 2,166 & 0.364 & 0.314 \\
\hline 7 & $\begin{array}{llll}380 & 207 & 36 & 153\end{array}$ & 10 & 1 & 0 & 700,270 & 2,174 & 0.310 & 0.230 \\
\hline 8 & $\begin{array}{llll}483 & 273 & 53 & 231\end{array}$ & 10 & 1 & 0 & 710,775 & 2,320 & 0.326 & 0.245 \\
\hline 9 & $\mid \begin{array}{llll}425 & 245 & 35 & 141\end{array}$ & 10 & 1 & 0 & 775,059 & 2,592 & 0.334 & 0.262 \\
\hline 10 & $\begin{array}{llll}196 & 118 & 17 & 68\end{array}$ & 10 & 1 & 0 & 522,015 & 1,182 & 0.226 & 0.190 \\
\hline
\end{tabular}

fewer changes on a single isosurface than the graph-based technique. The only places of significant change are the regions close to 0 or 1 ("flat" areas, subject to higher topological noise). The processing times are usually below 1 minute on a Pentium $43 \mathrm{Ghz}$ PC. GTCA requires approximately 2 minutes on a similar machine. All the brains we have tested so far take about the same time to process, and the amount of change is very similar from one brain to any other.

\section{Conclusions}

We have introduced in this paper an algorithm for propagating topology over scalar fields. It offers an efficient way to correct the topology of objects with a complex geometry, using all the available information of their membership function. It improves over previous methods by acting on all isovalues rather than a binary volume, and allowing non-spherical topologies. The technique was successfully tested on the problem of cortical segmentation to enforce a proper spherical topology on the white matter/gray matter interface. The propagation algorithm provides fast and reliable results, always very close to the original data. All isovalues have the correct topology, and the changes 
made on the membership function take into account the geometry of the entire image. The method can be applied to any kind of scalar image data, is fairly robust to noise, and guarantees exact results, in a single fast marching propagation step. Beyond correcting topology for membership functions, it also has potential applications to topology-preserving level set evolution and volumetric segmentation.

\section{References}

1. P.-L. Bazin and D. L. Pham. Topology smoothing for segmentation and surface reconstruction. In Proc. MICCAI'04, St Malo, Sept. 2004.

2. G. Bertrand. Simple points, topological numbers and geodesic neighborhood in cubic grids. Pattern Recognition Letters, 15(10):1003-1011, Oct. 1994.

3. S. Bischoff and L. Kobbelt. Sub-voxel topology control for level-set surfaces. Computer Graphics Forum, 22(3):273-280, 2003.

4. M. Couprie, F. Bezerra, and G. Bertrand. Topological operators for grayscale image processing. Journal of Electronic Imaging, 10(4):1003-1015, 2001.

5. X. Han, D. Pham, D. Tosun, M. Rettmann, C. Xu, and J. Prince. Cruise: Cortical reconstructiong using implicit surface evolution. NeuroImage, 23(3):997-1012, 2004.

6. X. Han, C. Xu, U. Braga-Neto, and J. L. Prince. Topology correction in brain cortex segmentation using a multiscale, graph-based algorithm. IEEE Trans. Med. Imaging:109-121, 21(2), 2002.

7. X. Han, C. Xu, and J. L. Prince. A topology preserving level set method for geometric deformable models. IEEE Trans. PAMI, 25(6):755-768, June 2003.

8. J. C. Hart. Morse theory for implicit surface modeling. In H.-C. Hege and K. Polthier, editors, Mathematical Visualization, pp 257-268. Springer-Verlag, Oct. 1998.

9. K. V. Leemput, F. Maes, D. Vandermeulen, and P. Suetens. Automated model-based tissue classification of mr images of the brain. IEEE Trans. on Med. Imaging, 18(10), 1999.

10. J.-F. Mangin, V. Frouin, I. Bloch, J. Regis, and J. Lopez-Krahe. From 3d magnetic resonance images to structural representations of the cortex topography using topology preserving deformations. J. Math. Imaging and Vision, 5:297-318, 1995.

11. D. L. Pham. Spatial models for fuzzy clustering. Computer Vision and Image Understanding, 84:285-297, 2001.

12. S. M. Resnick, A. F. Goldszal, C. Davatzikos, S. Golski, M. A. Kraut, E. J. Metter, R. N. Bryan, and A. B. Zonderman. One-year age changes in MRI brain volumes in older adults. Cerebral Cortex, 10(5):464-472, 2000.

13. F. Segonne, E. Grimson, and B. Fischl. Topological correction of subcortical segmentation. In Proc. MICCAI'03, Montreal, Nov. 2003.

14. J. Sethian. Level Set Methods and Fast Marching Methods. Cambridge University Press, 1999.

15. D. W. Shattuck and R. M. Leahy. Automated graph-based analysis and correction of cortical volume topology. IEEE Trans. on Med. Imaging, 20(11), Nov. 2001.

16. A. Szymczak and J. Vanderhyde. Extraction of topologically simple isosurfaces from volume datasets. In Proc. IEEE Visualization, pp 67-74, Seattle, Oct. 2003.

17. D. Tosun, M. Rettmann, D. Naiman, S. Resnick, M. Kraut, and J. Prince. Cortical reconstruction using implicit surface evolution: Accuracy and precision analysis. submitted to Neuroimage.

18. G. H. Weber, G. Scheuermann, and B. Hamann. Detecting critical regions in scalar fields. In Proc. EUROGRAPHICS - IEEE TCVG Symposium on Visualization, Grenoble, May 2003. 